\title{
Transcatheter aortic valve implantation procedure does not improve intraoperative left ventricular compliance.
}

\author{
Kosaku Toyota, Takashi Ota, Yasuhiro Koide \\ Department of anaesthesia, Shonan Kamakura General Hospital
}

\section{【Background 】}

Transcatheter Aortic Valve Implantation (TAVI) procedure for patients with aortic stenosis is expected to reduce left ventricular after load. Although many studies reported the effect of TAVI procedure on systolic function, the effect on perioperative diastolic function gets less attention. We presented significant LVEDP elevation just after SAPIEN valve deployment (ESA annual meeting 2015). However the mechanism of this LVEDP elevation is not clear.

\section{【Aim 】}

This retrospective observational study was aimed to investigate the change of ventricular compliance change.

\section{【Methods 】}

The study was approved by Institutional Review Board of Shonan Kamakura General Hospital (November,2015).

Subjects: Patients who underwent TAVI procedure using transfemoral approach with a balloon-expandable valve under general anesthesia

Study duration: June 1st 2014 July 31st 2015

Exclusion criteria: Cases with severe intraoperative complications which were needed for circulatory support devices. Cases which needed post dilatation for moderate to severe AR after deployment.

Time point of Measurements

Before aortic balloon valvuloplasty and after prosthetic valve deployment

Measurements (collecting data through procedure records.)

Left ventricular early and end diastolic pressure

Left ventricular transmitral inflow volume (derived using TEE)

Left ventricular compliance (calculated by dividing the left ventricular inflow volume by the left ventricular early to end diastolic pressure change)
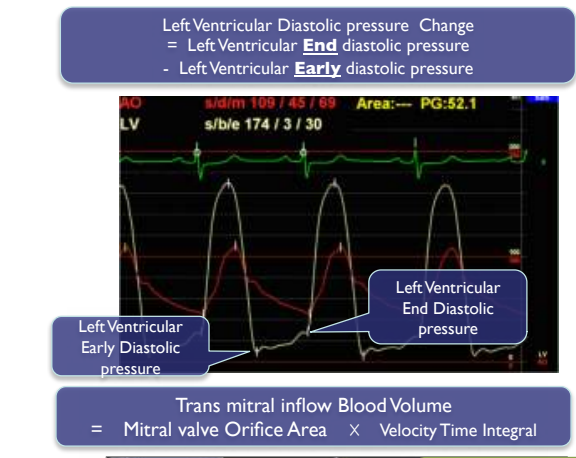

Statistical analysis:

Wilcoxon signed-rank test (P value less than 0.05 was defined as significant .)

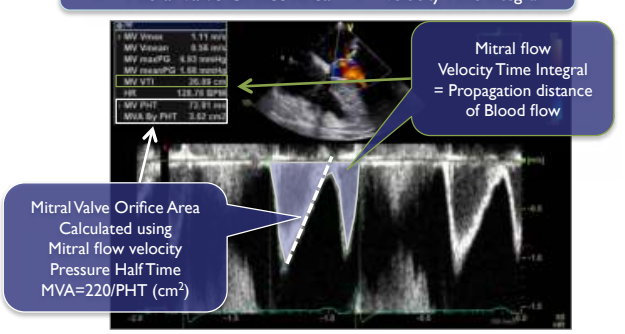

【Results】
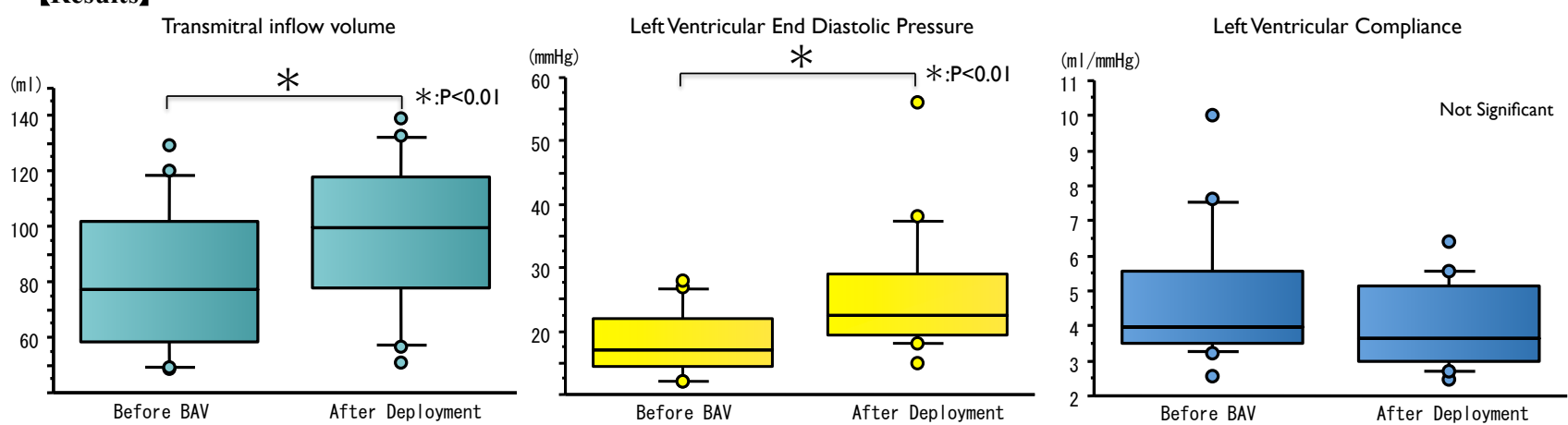

16 patients were enrolled in this study. The median value of left ventricular inflow volume was $77.3 \mathrm{ml}$ before balloon aortic valvuloplasty, and significantly increased to $99.7 \mathrm{ml}$ after prosthetic valve deployment.

The median LVEDP were $17.5 \mathrm{mmHg}$ and significantly increased to $30.0 \mathrm{mmHg}$ after prosthetic valve deployment.

The left ventricular compliance just before balloon aortic valvuloplasty and just after prosthetic valve deployment were $3.95 \mathrm{ml} / \mathrm{mmHg}$ and $3.62 \mathrm{ml} / \mathrm{mmHg}$, respectively. There was no significant difference between these left ventricular compliances.

【Conclusion】

TAVI procedure does not improve left ventricular compliance just after prosthetic valve deployment. This result indicates that the continuation of careful circulatory management is still required even after a valve deployment is successfully performed. 